\title{
Mortalidade por doenças do aparelho circulatório e fatores associados: estudo ecológico em municípios de Minas Gerais
}

\author{
Mortality due to circulatory system diseases and associated factors: an \\ ecological study in municipalities of Minas Gerais
}

\author{
Thiago Souza Barbosa ${ }^{1}$, Igor Vilela Brum ${ }^{2}$, Márcio José Martins Alves ${ }^{3}$, Maria Teresa \\ Bustamante Teixeira ${ }^{4}$
}

\begin{abstract}
RESUMO
Introdução: As doenças do aparelho circulatório (DAC) são responsáveis por 30\% das mortes no Brasil, além de gerar incapacidades e altos custos. Apesar disso, pouco se sabe sobre a relação entre a mortalidade por DAC e as condições de vida e assistência à saúde nos municípios brasileiros, especialmente em contextos regionais. Objetivo: Investigar a associação de variáveis de saúde e sociodemográficas com a mortalidade por DAC, em Minas Gerais (MG). Método: Estudo ecológico, a partir de dados secundários, tendo como unidade de análise 418 municípios de MG. A variável dependente foi a taxa de mortalidade por DAC e as variáveis de desfecho foram 32 indicadores de serviços de saúde e 22 indicadores demográficos e socioeconômicos. Realizou-se a análise bivariada dos dados, através do coeficiente de correlação de Pearson, seguida da análise fatorial e, por fim, da regressão linear múltipla. Resultados: As DAC ocasionaram 30,9\% da mortalidade total nos municípios analisados, observando-se associação positiva com número de consultórios de clínica básica, população urbana e internações por DAC no SUS; e negativa com valor médio por internação e despesa total com profissionais da saúde. Conclusão: Frente ao elevado número de municípios com pequena estrutura assistencial em MG, as ações de promoção à saúde e prevenção parecem ser as mais adequadas visando à redução das taxas de mortalidade por DAC. Também com este objetivo, os casos mais graves e as complicações devem ser manejados em centros de referência, contando com recursos materiais e profissionais adequados.
\end{abstract}

PALAVRAS-CHAVE: Doenças Cardiovasculares. Registros de Mortalidade.

\footnotetext{
${ }^{1}$ Faculdade de Medicina da Universidade Federal de Juiz de Fora.

2 Faculdade de Medicina da Universidade Federal de Juiz de Fora.

${ }^{3}$ Faculdade de Medicina da Universidade Federal de Juiz de Fora.

${ }^{4}$ Faculdade de Medicina da Universidade Federal de Juiz de Fora E-mail: teitabt@hotmail.com.
} 
Desigualdades em Saúde. Políticas Públicas.

\begin{abstract}
Introduction: Circulatory system diseases (CSD) are responsible for $30 \%$ of deaths in Brazil, as well as causing disabilities and high costs. Despite that, little is known about the relation between the mortality from CSD and the conditions of life and health care in Brazilian municipalities, especially in regional contexts. Objective: To investigate the association of health and sociodemographic variables with CSD mortality in Minas Gerais (MG). Methodology: Ecological study in which secondary data of 418 municipalities of MG were analyzed. The dependent variable was the mortality rate from CSD and the outcome variables consisted of 32 health services indicators and 22 demographic and socioeconomic indicators. A bivariate analysis of the data was performed by means of the Pearson's correlation coefficient, followed by factorial analysis, and finally, multiple linear regression. Results: CSD caused $30.9 \%$ of total mortality among the municipalities analyzed and were found to be positively associated with the number of general medicine clinics, urban population, and hospitalizations due to CSD. A negative association was observed with the average cost of hospitalizations and the total expenses with health professionals. Conclusion: In view of the high number of municipalities in MG having a scarce health care structure, actions aimed at promoting health and preventing disease appear to meet the most appropriate conditions for the reduction of CSD mortality rates. With this same purpose, the more severe cases and complications of CSD must be managed in reference centers, which dispose of adequate material and professional resources.
\end{abstract}

KEYWORDS: Cardiovascular Diseases. Mortality Registries. Health Inequalities. Public Policies.

\title{
INTRODUÇÃO
}

A partir de 1960, as doenças crônicas não transmissíveis (DCNT), incluindo as doenças do aparelho circulatório (DAC), passaram a representar a principal causa de mortalidade no Brasil. Até então, as doenças infecciosas eram responsáveis por quase metade do total de óbitos, sendo a primeira causa de morte em todas as regiões do país. ${ }^{1}$ Essa mudança no perfil epidemiológico brasileiro ocorreu devido às modificações na estrutura demográfica, caracterizada pela redução das taxas de fertilidade e aumento da expectativa de vida, com consequente aumento da proporção de idosos em relação aos demais grupos etários; às políticas de saúde pública, como os programas de medicina preventiva; e ao desenvolvimento econômico, incluindo a industrialização, a urbanização e as alterações do modo de vida a elas relacionadas. ${ }^{1,2}$

Com base nos registros do Sistema de Informações sobre Mortalidade (SIM), estima-se que as DAC sejam responsáveis por cerca de $30 \%$ de todas as mortes ocorridas no Brasil em um ano, ${ }^{3}$ com destaque para as doenças cerebrovasculares e isquêmicas 
do coração, que, juntas, representam mais de $60 \%$ dos óbitos por DAC. ${ }^{4}$ Esse grupo de doenças está relacionado com grande parte das internações e incapacidades, gerando alto custo para o Sistema Único de Saúde (SUS). ${ }^{2,5,6}$

A distribuição das DAC, incluindo a exposição a seus fatores de risco e sua morbimortalidade, não se dá de maneira aleatória ou homogênea, estando relacionada às condições de vida de cada grupo populacional. ${ }^{7,8}$ Maiores taxas de mortalidade por DAC nas regiões com menores níveis socioeconômicos e de escolaridade e maiores índices de desigualdade social já foram amplamente demonstrados na literatura internacional. ${ }^{9-12}$ No Brasil, apenas, recentemente, maior atenção tem sido dada a como aspectos socioeconômicos, educacionais e demográficos influenciam a mortalidade por DAC, tendo-se observado, de modo similar, maiores taxas nos estratos sociais mais vulneráveis, com baixa renda e escolaridade. ${ }^{8,13-15}$

A maioria dos estudos nacionais com essa temática teve, no entanto, como unidade de análise, capitais de estados e municípios de médio ou grande porte (mais de 100 mil habitantes), de forma que a realidade em cidades menores ainda é pouco conhecida. ${ }^{8}$ Além disso, a maior parte das pesquisas sobre DAC em contextos regionais utilizaram populações do Rio de Janeiro e de São Paulo, ${ }^{16,17}$ de modo que Minas Gerais (MG) ainda tem participação discreta nesse cenário de estudos. ${ }^{18}$ Observa-se, portanto, a necessidade de se ampliar o enfoque dado ao estudo das DAC em diferentes realidades contextuais, levando-se em conta determinantes demográficos, sociais e de acesso à saúde em diferentes espaços geográficos brasileiros.

O Plano de Ações Estratégicas para o Enfrentamento das DCNT, elaborado em 2011, reúne as principais metas e compromissos para prevenção e gerenciamento das DAC e outras doenças crônicas no Brasil. Entre tais estratégias, destaca-se a expansão da Atenção Básica em Saúde (ABS), visando ações de promoção e vigilância em saúde, prevenção, assistência e acompanhamento dos usuários. ${ }^{19} \mathrm{~A}$ ABS abrange cerca de $60 \%$ da população brasileira e, embora tenha expandido na última década, persistem importantes diferenças entre os municípios em relação à cobertura, acesso e oferta de cuidados, bem como nos investimentos em saúde de maneira geral. ${ }^{20}$ Há pouca clareza, entretanto, em relação a como essas diferentes realidades assistenciais têm afetado a carga das DAC nos municípios.

Este estudo teve por objetivo investigar a associação entre a mortalidade por 
DAC e indicadores socioeconômicos, demográficos, de cobertura e de acesso aos serviços de saúde nos municípios de MG, buscando identificar entre diversos fatores, aqueles com maior impacto nas taxas de mortalidade.

\section{MATERIAL E MÉTODOS}

Estudo ecológico cuja unidade de análise foram os municípios do estado de MG. A variável dependente foi a taxa de mortalidade por DAC, considerando os óbitos de residentes de cada município classificados no capítulo IX da CID-10. Para o cálculo das taxas de mortalidade por DAC, obtiveram-se dados entre o período de 2006 e 2010, fornecidos pelo Sistema de Informação de Mortalidade (SIM), no sítio do DATASUS do Ministério da Saúde. ${ }^{21}$ Considerou-se como numerador a média do número de óbitos no período de 5 anos, por faixa etária e como denominador a população da mesma faixa etária de cada município projetada pelo Instituto Brasileiro de Geografia e Estatística (IBGE) para 2008, numa razão expressa para cada 100.000 habitantes. As taxas foram padronizadas pelo método direto para a idade, de acordo com a população padrão mundial. $^{22}$

Foram consideradas inicialmente 54 variáveis preditoras, divididas a priori em duas categorias: 32 Indicadores de serviços de saúde (estrutura, processo e cobertura da Estratégia Saúde da Família (ESF)/ABS), obtidos de fontes do Ministério da Saúde, a saber: SIM, Sistema de Informação da Atenção Básica (SIAB), Cadastro Nacional de Estabelecimentos de Saúde (CNES), Sistema de Informações Hospitalares Descentralizado (SIHD) e Cadernos de Informação de Saúde ${ }^{21}$ dispostos na tabela 1; e 22 indicadores demográficos e socioeconômicos, obtidos de fontes do IBGE $^{23}$ e do Programa das Nações Unidas para o Desenvolvimento (PNUD), ${ }^{24}$ dispostos na tabela 2.

Tabela 1- Indicadores de serviços de saúde analisados.

\begin{tabular}{llll}
\hline Indicador & Unidade & Fonte & Ano \\
\hline População cadastrada na ESF & \% população total & SIAB & $2006-10$ \\
Cobertura pela ABS & \% população total & CNES & 2008 \\
Agentes comunitários de saúde & Profissionais/10000 hab. & CNES & 2008 \\
Enfermeiras (os) & Profissionais/10000 hab. & CNES & 2008 \\
\hline
\end{tabular}




\begin{tabular}{|c|c|c|c|}
\hline & & & (conclusão) \\
\hline Indicador & Unidade & Fonte & Ano \\
\hline Médicos (as) alocados na APS & Profissionais/10000 hab. & CNES & 2008 \\
\hline Médicos totais & Profissionais/10000 hab. & CNES & 2008 \\
\hline Médicos cardiologistas & Profissionais/10000 hab. & CNES & 2008 \\
\hline Profissionais de saúde c/ nível superior & Profissionais/10000 hab. & CNES & 2008 \\
\hline Consultórios médicos totais & Total/10000 hab. & CNES & 2008 \\
\hline Consultórios médicos de clínica básica & Total/10000 hab. & CNES & 2008 \\
\hline Consultórios médicos e não médicos & Total/10000 hab. & CNES & 2008 \\
\hline Consultórios de urgência & Total/10000 hab. & CNES & 2008 \\
\hline Leitos totais de internação & Total/10000 hab. & SIHD & 2009 \\
\hline Leitos SUS de internação & Total/10000 hab. & SIHD & 2009 \\
\hline Produção ambulatorial do SUS & Número consultas/hab. & SIHD & 2008 \\
\hline Despesa total com saúde & Valor em reais/hab. & SIOPS & 2008 \\
\hline Despesa total c/ profissionais da saúde & Valor em reais/hab. & SIOPS & 2008 \\
\hline Despesa hospitalar total & Valor em reais/hab. & SIOPS & 2008 \\
\hline Despesa com internações & Valor reais/internação & SIOPS & 2008 \\
\hline Despesa com internações p/ DCV & Valor em reais/hab. & SIOPS & 2008 \\
\hline $\begin{array}{l}\text { Procedimentos hospitalares do SUS } \\
\text { para tratamento de DCV }\end{array}$ & Total/10000 hab. & SIHD & 2008 \\
\hline Tempo de internação no SUS para DCV & Média dias/internação & SIHD & 2008 \\
\hline Internações por DAC no SUS & Total/10000 hab. & SIHD & 2008 \\
\hline Internações por AVC no SUS & Total/10000 hab. & SIHD & 2008 \\
\hline Internações por IAM no SUS & Total/10000 hab. & SIHD & 2008 \\
\hline Internações por DCV - urgência no SUS & Total/10000 hab. & SIHD & 2008 \\
\hline Distância da regional de saúde & Quilômetros & IBGE & 2009 \\
\hline Aparelhos de raio-X & Equipamentos/10000 hab. & CNES & 2008 \\
\hline ECG & Equipamentos/10000 hab. & CNES & 2008 \\
\hline Monitor de ECG & Equipamentos/10000hab. & CNES & 2008 \\
\hline DAE & Equipamentos/10000 hab. & CNES & 2008 \\
\hline Reanimador pulmonar - AMBU & Equipamentos/10000 hab. & CNES & 2008 \\
\hline \multicolumn{4}{|c|}{$\begin{array}{l}\text { ESF: Estratégia Saúde da Família; SIAB: Sistema de Informação da Atenção Básica; ABS: Atenção Básica à Saúde; } \\
\text { CNES: Cadastro Nacional de Estabelecimentos de Saúde; hab.: habitantes; APS: Atenção Primária à Saúde; SIHD: } \\
\text { Sistema de Informações Hospitalares Descentralizado; SUS: Sistema Único de Saúde; SIOPS: Sistema de } \\
\text { Informações sobre Orçamentos Públicos em Saúde; DCV: doenças cardiovasculares; DAC: doenças do aparelho } \\
\text { circulatório; ACV: acidente vascular cerebral; IAM: infarto agudo do miocárdio; ECG: eletrocardiograma; DAE: } \\
\text { desfibrilador automático externo. }\end{array}$} \\
\hline
\end{tabular}

Fonte: elaborada pelos autores 
Tabela 2- Indicadores demográficos e socioeconômicos analisados.

\begin{tabular}{|c|c|c|c|}
\hline Indicador & Unidade & Fonte & Ano \\
\hline Desemprego $\geq 16$ anos & \% população total & IBGE & 2010 \\
\hline Trabalho infantil (10 a 15 anos) & \% população total & IBGE & 2010 \\
\hline Analfabetismo ( $\geq 15$ anos) & \% população total & IBGE & 2010 \\
\hline Mortalidade infantil & $\begin{array}{l}\text { Óbitos em }<1 \text { ano/1000 } \\
\text { nascidos vivos. }\end{array}$ & $\begin{array}{l}\text { SIM } \\
\text { SINASC }\end{array}$ & 2007-10 \\
\hline $\begin{array}{l}\text { População } \geq 15 \text { anos com Ensino } \\
\text { Médio completo ou maior }\end{array}$ & \% população total & IBGE & 2010 \\
\hline População com renda < 1/4 SM & \% população total & IBGE & 2010 \\
\hline População com renda < 1/2 SM & \% população total & IBGE & 2010 \\
\hline PIB per capita municipal & Valor em reais & PNUD & 2008 \\
\hline Renda média domiciliar per capita & Valor em reais & IBGE & 2010 \\
\hline Razão de renda & $\begin{array}{l}\text { Renda } 20 \%+\text { ricos/ renda } \\
20 \%+\text { pobres }\end{array}$ & IBGE & 2010 \\
\hline População total do município & População total & IBGE & 2008 \\
\hline População urbana & \% população total & IBGE & 2010 \\
\hline População masculina $\geq 50$ anos & $\%$ homens com $\geq 50$ anos & IBGE & 2010 \\
\hline IDH municipal & 0 a 1 & PNUD & 2010 \\
\hline IDH municipal - educação & 0 a 1 & PNUD & 2010 \\
\hline IDH municipal - longevidade & 0 a 1 & PNUD & 2010 \\
\hline IDH municipal - renda & 0 a 1 & PNUD & 2010 \\
\hline Índice de GINI & 0 a 1 & PNUD & 2010 \\
\hline Domicílios com água encanada & $\%$ dos domicílios & IBGE & 2000 \\
\hline Domicílios com poço ou nascente & $\%$ dos domicílios & IBGE & 2000 \\
\hline Domicílios sem instalação sanitária & $\%$ dos domicílios & IBGE & 2000 \\
\hline
\end{tabular}

Fonte: elaborada pelos autores

O estado de MG possui 853 municípios. Como critério para a seleção da amostra considerou-se a qualidade dos dados, admitindo-se para inclusão do município no estudo uma proporção máxima de $10 \%$ de causas de óbito mal definidas, ${ }^{11}$ sendo que esse processo resultou na seleção final de 418 municípios.

Para o cálculo dos indicadores e confecção das tabelas, foi utilizado o software 
Excel e para as análises de correlação e regressão múltipla foi utilizado o software Statistical Package for the Social Sciences (SPSS) versão 17. Inicialmente, realizou-se a apreciação das distribuições de frequência univariadas para cada indicador, com o intuito de se detectar eventuais pontos discrepantes extremos, que foram eliminados. Em seguida, a distribuição dos dados em torno da média foi analisada, sendo que os indicadores assimétricos foram submetidos a transformações algébricas adequadas para que se tornassem mais compatíveis com as suposições de normalidade. ${ }^{25}$

Procedeu-se com a análise bivariada dos dados, através do coeficiente de correlação de Pearson, sendo selecionadas para a análise multivariada apenas as variáveis preditoras, que obtiveram correlação significativa com a variável dependente a um nível $\alpha=0,05$. Na análise de resíduos, foram eliminados os pontos que estavam além de três desvios padrão da distribuição aproximadamente normal dos dados.

$\mathrm{Na}$ análise multivariada, procedeu-se à análise fatorial (AF) para cada uma das duas categorias de indicadores, com objetivo de reduzir o número inicial de variáveis. $\mathrm{Na} A F$, foram gerados fatores principais, ou seja, variáveis artificiais projetadas para maximizar o modelo, partindo do princípio de que um número bem menor de fatores constitui as principais dimensões do problema, abandonando-se os demais sob a hipótese de que sua contribuição, para explicar a variabilidade total dos dados pode ser considerada residual. Para aumentar a interpretabilidade dos fatores, adotou-se o método de rotação ortogonal Varimax. Foram retidos os fatores que preencheram os seguintes critérios: autovalor maior que 1 e quantidade de fatores que explicassem pelo menos $70 \%$ da variância total dos dados. Consideraram-se as variáveis como pertencentes a um fator, quando o coeficiente de correlação da variável com o fator foi maior que 0,600 em valores absolutos. ${ }^{25}$

Tendo como variável dependente a mortalidade por DAC e, partindo do conjunto de variáveis indicadas pela $A F$, obteve-se o modelo inicial com uma variável de cada fator da AF, de acordo com o critério de autovalor. Este procedimento de escolha das variáveis independentes (explicativas da mortalidade), guiado pela $A F$, visa evitar problemas de multicolinearidade (correlação das variáveis independentes entre si), pois as mesmas devem ser descorrelacionadas.

Procedeu-se à construção do modelo final, explicativo da mortalidade por DAC, através de regressão linear múltipla (RLM). Nessa fase, foram retiradas uma a uma as 
variáveis que não apresentaram significância, e mantidas as variáveis mais importantes para a discussão do modelo final. ${ }^{25}$

O projeto foi submetido ao Comitê de Ética em Pesquisa Humana da Universidade Federal de Juiz de Fora, sendo analisado e aprovado sob o parecer número 356.920 .

\section{RESULTADOS}

Entre os 418 municípios analisados, 29,9\% possuíam população inferior a 5 mil habitantes; 28,0\%, entre 5 e 10 mil; 21,5\%, entre 10 e 20 mil; 15,6\%, entre 20 e 100 mil; e 4,3\%, acima de 100 mil. A média populacional foi de 29178 habitantes e a mediana, 7770,5. Ao todo, 211 (50,5\%) municípios tinham mais de $25 \%$ da população, residindo em área rural e em 55 (13,2\%), a população rural correspondia à maioria. A cobertura média da ESF foi $81,2 \%$, sendo maior entre os municípios de até 5 mil habitantes, nos quais a média da população coberta foi 95,3\%.

A mortalidade proporcional por DAC no período representou $30,9 \%$ da mortalidade total dos municípios. A taxa média de mortalidade por DAC foi 150,1 óbitos/ano/100000 habitantes e a mediana, 149,23. A menor e a maior taxa foram 52,3 e 273,9 , respectivamente.

As taxas de mortalidade por DAC correlacionaram-se na análise bivariada com 37 indicadores, sendo 23 da categoria de serviços de saúde e 14 da categoria de indicadores demográficos e socioeconômicos, estando os mesmos descritos nas tabelas 3 e 4.

Tabela 3- Correlação da taxa de mortalidade por DAC padronizada com indicadores de serviços de saúde. Minas Gerais, 2006 - 2010.

\begin{tabular}{lccc}
\hline Indicador & Correlação Pearson (R) & $\mathbf{R}^{\mathbf{2}}$ & Significância \\
\hline Despesa total c/ profissionais da & $-0,226$ & 0,051 & 0,000 \\
saúde & & & \\
Despesa total com saúde & $-0,186$ & 0,035 & 0,000 \\
Cobertura pela ABS & $-0,186$ & 0,035 & 0,000 \\
Valor médio por internação & $-0,168$ & 0,028 & 0,001 \\
\hline
\end{tabular}




\begin{tabular}{|c|c|c|c|}
\hline Indicador & Correlação Pearson (R) & $\mathbf{R}^{2}$ & $\begin{array}{l}\text { (conclusão } \\
\text { Significância }\end{array}$ \\
\hline População cadastrada na ESF & $-0,158$ & 0,025 & 0,001 \\
\hline Agentes comunitários de saúde & $-0,114$ & 0,013 & 0,021 \\
\hline DAE & 0,104 & 0,011 & 0,035 \\
\hline Aparelhos de raio- $X$ & 0,104 & 0,011 & 0,035 \\
\hline Médicos cardiologistas & 0,110 & 0,012 & 0,028 \\
\hline Despesa com internações p/ DCV & 0,117 & 0,014 & 0,017 \\
\hline Consultórios médicos e não médicos & 0,153 & 0,023 & 0,002 \\
\hline Produção ambulatorial do SUS & 0,178 & 0,032 & 0,000 \\
\hline Despesa hospitalar total & 0,189 & 0,036 & 0,000 \\
\hline Consultórios médicos totais & 0,207 & 0,043 & 0,000 \\
\hline Monitor de ECG & 0,216 & 0,047 & 0,000 \\
\hline Leitos totais de internação & 0,229 & 0,052 & 0,000 \\
\hline Leitos SUS de internação & 0,230 & 0,053 & 0,000 \\
\hline Internações por AVC no SUS & 0,233 & 0,054 & 0,000 \\
\hline $\begin{array}{l}\text { Consultórios médicos de clínica } \\
\text { básica }\end{array}$ & 0,247 & 0,061 & 0,000 \\
\hline $\begin{array}{l}\text { Internações por DCV - urgência no } \\
\text { SUS }\end{array}$ & 0,271 & 0,073 & 0,000 \\
\hline $\begin{array}{l}\text { Procedimentos hospitalares do SUS } \\
\text { para tratamento de DCV }\end{array}$ & 0,287 & 0,082 & 0,000 \\
\hline Internações por DAC no SUS & 0,293 & 0,086 & 0,000 \\
\hline
\end{tabular}

ESF: Estratégia Saúde da Família; ABS: atenção básica à saúde; DAE: desfibrilador automático externo; DCV: doenças cardiovasculares; DAC: doenças do aparelho circulatório; SUS: Sistema Único de Saúde; ECG: eletrocardiograma; ACV: acidente vascular cerebral.

Fonte: elaborada pelos autores

Tabela 4- Correlação da taxa de mortalidade por DAC padronizada com indicadores demográficos e socioeconômicos. Minas Gerais, 2006 - 2010.

\begin{tabular}{llll}
\hline Indicador & Correlação Pearson (R) & $\mathbf{R}^{\mathbf{2}}$ & Significância \\
\hline Domicílios com poço ou nascente & $-0,248$ & 0,062 & 0,000 \\
Analfabetismo ( $\geq 15$ anos) & $-0,172$ & 0,030 & 0,000 \\
População sem instrução ou Ensino & $-0,161$ & 0,026 & 0,001 \\
Fundamental incompleto & & & \\
Domicílios sem instalação sanitária & $-0,108$ & 0,012 & 0,031 \\
\hline
\end{tabular}




\begin{tabular}{|c|c|c|c|}
\hline Indicador & Correlação Pearson (R) & $\mathbf{R}^{2}$ & $\begin{array}{l}\text { (conclusão) } \\
\text { Significância }\end{array}$ \\
\hline $\begin{array}{l}\text { População com renda }<1 / 4 \text { salário } \\
\text { mínimo }\end{array}$ & $-0,100$ & 0,010 & 0,041 \\
\hline IDH municipal & 0,097 & 0,009 & 0,048 \\
\hline IDH municipal - educação & 0,114 & 0,013 & 0,020 \\
\hline População masculina $\geq 50$ anos & 0,124 & 0,015 & 0,011 \\
\hline Desemprego $\geq 16$ anos & 0,127 & 0,016 & 0,010 \\
\hline $\begin{array}{l}\text { População com Ensino Médio } \\
\text { completo ou }+(e m \geq 15 \text { anos })\end{array}$ & 0,195 & 0,038 & 0,000 \\
\hline População total do município & 0,210 & 0,044 & 0,000 \\
\hline Domicílios com água encanada & 0,247 & 0,061 & 0,000 \\
\hline População urbana & 0,251 & 0,063 & 0,000 \\
\hline
\end{tabular}

IDH: Índice de Desenvolvimento Humano

Fonte: elaborada pelos autores

A AF resultou em 6 fatores principais na categoria de indicadores de serviços de saúde, capazes de explicar 73,5\% da variância total dos dados, e 3 fatores principais na categoria de indicadores demográficos e socioeconômicos, explicativos de $72,4 \%$ da variância dos dados. O resultado da AF e os indicadores aglutinados em cada fator estão descritos na tabela 5.

Tabela 5- Resultado da análise fatorial: fatores principais e variáveis aglutinadas em cada fator para as categorias de indicadores de serviços de saúde e indicadores demográficos e socioeconômicos.

\begin{tabular}{ll}
\hline Fatores & Indicadores de serviços de saúde \\
\hline 1 & Internações por DAC no SUS*; Procedimentos hospitalares do SUS para tratamento \\
& de DCV; Internações por DAC - urgência no SUS \\
2 & Leitos totais de internação; Leitos SUS de internação*; Aparelhos de raio-X; \\
& Monitor de ECG; DAE \\
3 & População cadastrada na ESF*; Cobertura pela ABS; Agentes comunitários \\
& Consultórios médicos totais; Consultórios médicos de clínica básica*; Consultórios \\
& médicos e não médicos
\end{tabular}


(conclusão)

\section{Fatores Indicadores de serviços de saúde}

\begin{tabular}{ll}
\hline 5 & Despesa hospitalar total; Valor médio por internação*; Despesa com internações \\
& para DCV \\
& Despesa total com saúde; Despesa total com profissionais da saúde* \\
\hline Fatores & Indicadores demográficos e socioeconômicos \\
\hline 1 & População urbana*; População total do município; Analfabetismo ( $\geq 15$ anos); \\
& População com Ensino Médio completo ou + (em $\geq 15$ anos); População sem \\
& instrução ou Ensino Fundamental incompleto; População com renda < 1/4 salário \\
& mínimo; Domicílios com água encanada; Domicílios sem instalação sanitária; \\
& Domicílios com poço ou nascente. \\
& Desemprego $\geq 16$ anos; População masculina $\geq 50$ anos* \\
& IDH municipal*; IDH municipal - educação \\
\hline $\begin{array}{l}\text { DAC: doenças do aparelho circulatório; SUS: Sistema Único de Saúde; DCV: doenças cardiovasculares; ECG: } \\
\text { eletrocardiograma; DAE: desfibrilador automático externo; ESF: Estratégia Saúde da Família; ABS: atenço básica } \\
\text { à saúde; IDH: Índice de Desenvolvimento Humano. * Variáveis selecionadas para a Regressão Linear Múltipla. }\end{array}$
\end{tabular}

Fonte: elaborada pelos autores

Para a RLM, selecionou-se uma variável de cada fator principal (tabela 5), considerando, sempre que possível, as variáveis de maior coeficiente fatorial e maior coeficiente de correlação de Pearson. A RLM mostrou correlação positiva da taxa de mortalidade por DAC com os indicadores: consultórios médicos de clínica básica, porcentagem da população urbana e internações por DAC no SUS; e correlação negativa com: valor médio por internação e despesa total com profissionais da saúde. Houve ainda, embora com significância limítrofe, correlação negativa da mortalidade por DAC com a porcentagem da população cadastrada na ESF e positiva com a proporção da população masculina com mais de 50 anos. Os resultados da RLM estão detalhados na tabela 6 , apresentada na próxima página. 
Tabela 6 - Resultados da Regressão Linear Múltipla da taxa de mortalidade por DAC padronizada com os indicadores selecionados pela análise fatorial. Minas Gerais, 2006 - 2010.

\begin{tabular}{lll}
\hline Indicador & T & Significância \\
\hline Valor médio por internação & $-2,511$ & 0,012 \\
Despesa total com profissionais da saúde & $-2,364$ & 0,019 \\
População cadastrada na ESF & $-1,654$ & 0,099 \\
Leitos SUS de internação & 0,484 & 0,629 \\
IDH municipal & 0,635 & 0,526 \\
População masculina $\geq 50$ anos & 1,716 & 0,087 \\
Consultórios médicos de clínica básica & 3,496 & 0,001 \\
População urbana & 3,593 & 0,000 \\
Internações por DAC no SUS & 4,086 & 0,000
\end{tabular}

ESF: Estratégia Saúde da Família; SUS: Sistema Único de Saúde; IDH: índice de desenvolvimento humano; DAC: doenças do aparelho circulatório.

Fonte: elaborada pelos autores

\section{DISCUSSÃO}

O estudo descreveu a mortalidade média por DAC e fatores associados nos municípios de MG. Entre os principais resultados, destacam-se a proporção das DAC entre as causas de mortalidade, correspondendo a aproximadamente $30 \%$ do total de óbitos; as diferenças expressivas nas taxas de mortalidade por DAC entre os municípios, sendo superior a 5 vezes, quando comparadas as maiores e menores taxas; e a identificação de alguns fatores relacionados à assistência à saúde, como valor da internação e despesa com profissionais da saúde, que possam contribuir para tais diferenças observadas.

O estado de MG possui características demográficas próprias, que tornam os resultados deste estudo particulares em relação a outras investigações nacionais. De um total de 853 municípios, 824 (96,6\%) possuem população inferior a 100 mil habitantes e 675 (79,1\%), inferior a 20 mil. ${ }^{26}$ A maioria dessas localidades enfrenta dificuldades para garantir o atendimento adequado à população, visto que os desafios vão, desde a estrutura física assistencial deficiente e a inexistência de serviços de atenção secundária 
e/ou terciária para complementação diagnóstica e terapêutica, até a limitação para qualificar e reter profissionais de saúde e de gestão.

Nesse contexto, considerando, ainda, que o tratamento das complicações das DAC é frequentemente dispendioso e dependente de tecnologias sofisticadas específicas, ${ }^{28}$ as medidas de promoção à saúde, prevenção e controle das DAC mostramse como alternativas mais viáveis, visando reduzir a demanda por hospitalizações. Com esse objetivo, as ações desenvolvidas na $A B S$, ao permitirem a aproximação dos serviços de saúde com os indivíduos, seu coletivo e suas interações sociais, reúnem as condições mais favoráveis para promover mudanças de comportamentos e hábitos de vida saudáveis. ${ }^{28}$

No Rio Grande do Sul, por exemplo, a cobertura da ESF, principal ferramenta de reorganização da $A B S$, mostrou ser um fator protetor maior para a mortalidade por DAC nos municípios com menor número de habitantes. ${ }^{29}$ Esse aspecto merece atenção especial em MG diante da composição demográfica supracitada. Neste estudo, embora com significância limítrofe, foram identificadas menores taxas de mortalidade por DAC nos municípios com maior cobertura da ESF. Levando-se em conta que a ampliação da ABS é um processo relativamente recente e ainda em implementação, espera-se que, com o passar dos anos, o impacto da ABS na mortalidade por DAC se revele de forma mais consolidada.

Se, por um lado, a promoção de comportamentos e estilos de vida saudáveis deva ser central no manejo das DAC, por outro, a incorporação de novas tecnologias e o acesso a procedimentos de média e alta complexidade também são componentes fundamentais na garantia da integralidade da assistência. ${ }^{19}$ No entanto, o que se observa em MG é que a maioria dos pequenos municípios não dispõe desses serviços ou de centros de referência e encontra-se em situação de grande dependência externa em relação aos poucos locais, que contam com serviços de atenção secundária e/ou terciária. O acesso a esses serviços é importante e, possivelmente, se revela, neste estudo, pelas menores taxas de mortalidade por DAC em função do valor médio por internação. Isso ocorre pelo fato de os serviços de referência, em geral, estarem relacionados com internações, que demandam maiores custos, porém essenciais na garantia de assistência qualificada nos casos de complicações e gravidade.

A maior mortalidade por DAC em função do número de internações por DAC no 
SUS, observada neste estudo, é um segundo elemento que corrobora a importância dos serviços de referência, que dispõe de recursos materiais e humanos adequados, no tratamento das complicações das DAC. A internação dos pacientes por si só, quando em condições subótimas de cuidado, possivelmente não é resolutiva, refletindo em maior mortalidade. Um exemplo de estratégia, nesse sentido, consiste na criação de unidades de atendimento ao acidente vascular encefálico nos hospitais de referência, visando a assistência qualificada e o cuidado multiprofissional. ${ }^{19}$ Este último aspecto, inclusive, parece ser uma estratégia fundamental para reduzir óbitos por DAC, na medida em que o gasto médio com profissionais de saúde se revelou, neste estudo, como um aspecto protetor da mortalidade.

Além disso, considerando que a prevenção e controle das DAC são realizados essencialmente na $A B S$, a maior mortalidade por DAC em função do número de internações, conforme observado, também pode ser interpretada como um reflexo de dificuldades assistenciais mais amplas, visto que altas taxas de internação podem refletir um pior desempenho do sistema de saúde. ${ }^{30}$

Como os pacientes com DAC, de um modo geral, não se curam, os benefícios das medidas de controle são pequenos quando comparados aos benefícios da promoção à saúde e prevenção de doença. ${ }^{31}$ Dessa forma, o fato de um maior número de consultórios médicos, onde, em geral, são realizadas as medidas de controle das DAC, ter se mostrado associado a maior mortalidade por DAC, pode indicar que, assim como as internações hospitalares, o controle ambulatorial, muitas vezes, não é resolutivo, reforçando o fato de que a redução da mortalidade por DAC encontra seu maior potencial nas ações de prevenção e controle desse grupo de doenças.

A grande migração de pacientes e, muitas vezes, de dados, para os grandes centros, como consequência da situação de dependência dos pequenos municípios em MG, pode interferir no registro de informações, fazendo com que a taxa de mortalidade por DAC encontre-se aumentada nos maiores centros urbanos. Apesar disso, a urbanização, por si só, está classicamente associada a uma maior prevalência de DAC. ${ }^{32}$ Um dos motivos para tal seria a maior exposição a fatores de risco, como HAS e DM, nas populações urbanas em comparação com as rurais. ${ }^{33,34}$ Levando-se em conta que a urbanização é um fenômeno crescente, é de se esperar que a demanda por atendimento das DAC aumente como o deslocamento das populações, impondo, de forma constante, 
novos desafios, para o setor da saúde e a sociedade em geral.

Embora a qualidade das informações no SIM tenha progressivamente melhorado, um fator limitante deste estudo consiste na utilização de dados secundários, devido à possibilidade de subregistro ou de preenchimento incorreto da declaração de óbito. ${ }^{35}$ Além disso, embora a análise de dados de municípios de pequeno porte tenha contribuído para a originalidade deste estudo, pode ter havido nesses casos, em função do pequeno número de óbitos, flutuação aleatória dos dados de mortalidade, fato minimizado pela análise da média dos óbitos ocorridos em 5 anos. Por outro lado, uma vantagem deste estudo, por meio do tratamento estatístico adequado, foi a eliminação de outliers e fatores de confusão que, inicialmente, mostraram-se associados à mortalidade por DAC, identificando aqueles que, de fato, possuem maior impacto nas taxas de mortalidade.

O desfecho analisado neste estudo foi unicamente a mortalidade por DAC, de forma que novas pesquisas são necessárias para avaliar outros aspectos relacionados às DAC, como o grau de limitação e incapacidade gerados e os impactos emocionais e econômicos para famílias, comunidades e sociedade em geral. Além disso, pesquisas longitudinais são necessárias para que, além de se identificar as origens das diferenças de mortalidade nos municípios, possa monitorar o impacto das medidas de enfrentamento e as mudanças ao longo do tempo.

\section{CONCLUSÃO}

As mudanças epidemiológicas observadas na população brasileira impõem novos desafios no manejo das DAC. Frente a essa realidade, as associações encontradas neste estudo fornecem subsídios acerca da importância de se direcionar os recursos de financiamento em saúde para ações de promoção e prevenção das DAC, mais efetivas na redução da mortalidade se comparadas ao controle ambulatorial e/ou hospitalar. Isso ganha importância ainda maior no contexto de MG, onde predominam municípios com pequena infraestrutura assistencial. Por outro lado, na medida em que as complicações são inerentes à história natural da maioria das DAC, o acesso a serviços de referência especializados deve ser garantido, visto que o valor médio das internações e gasto com profissionais de saúde se mostraram relacionados com menores taxas de 
mortalidade.

\section{REFERÊNCIAS}

1. Prata PR. The epidemiologic transition in Brazil. Cad. Saúde Pública. 1992 jun.; $8(2): 168-75$.

2. Schramm JMA, Oliveira AF, Leite IC, Valente JG, Gadelha AMJ, Portela MC, et al. Transição epidemiológica e o estudo de carga de doença no Brasil. Ciênc. Saúde Coletiva. 2004; 9(4):897-908.

3. Malta DC, Moura LD, Prado RR, Escalante JC, Schmidt MI, Duncan BB. Mortalidade por doenças crônicas não transmissíveis no Brasil e suas regiões, 2000 a 2011. Epidemiol. Serv. Saúde. 2014 dez.; 23(4):599-608.

4. Perisse G, Medronho RA, Escosteguy CC. Espaço urbano e a mortalidade por doença isquêmica do coração em idosos no Rio de Janeiro. Arq. Bras. Cardiol. 2010; 94(4):463-71.

5. Rede Interagencial de Informação para a Saúde. Indicadores básicos para a saúde no Brasil: conceitos e aplicações [Internet]. Brasília: Organização Pan-Americana da Saúde; 2008 [Citado 2012 fev. 05]. Disponível em: <http://tabnet.datasus.gov.br/tabdata/livroidb/2ed/indicadores.pdf $>$.

6. Teich V, Araujo DV. Estimativa de custo da síndrome coronariana aguda no Brasil. Rev. Bras. Cardiol. 2011 mar.; 24(2):85-94.

7. Lahelma E, Martikainen $P$, Laaksonen $M$, Aittomäki A. Pathways between socioeconomic determinants of health. J. Epidemiol. Community Health. 2004 apr. $1 ; 58(4): 327-32$.

8. Ishitani LH, Franco GD, Perpétuo IH, França E. Desigualdade social e mortalidade precoce por doenças cardiovasculares no Brasil. Rev. Saúde Pública. 2006 ago. 1; 40(4):684-91.

9. Ross NA, Wolfson MC, Dunn JR, Berthelot JM, Kaplan GA, Lynch JW. Relation between income inequality and mortality in Canada and in the United States: cross sectional assessment using census data and vital statistics. BMJ. 2000 apr. 1; 320(7239):898-902.

10. Gupta R, Deedwania PC, Sharma K, Gupta A, Guptha S, Achari V, et al. Association of Educational, Occupational and Socioeconomic Status with Cardiovascular Risk Factors in Asian Indians: A Cross-Sectional Study. PLoS One. 2012; 7(8):1-10.

11. Le C, Chongsuvivatwong V, Geater A. Contextual socioeconomic determinants of cardiovascular risk factors in rural south-west China: a multilevel analysis. BMC Public Health. 2007 may 5; 7(1):72.

12. Yoon JM, Kim JH, Suh BS, Park SM. Educational disparities in distribution of cardiovascular risk factors and quality of care in Korean adults: Korean National Health and Nutrition Survey IV. Korean J. Fam. Med. 2013 jan. 1; 34(1):27-35. 
13. Lessa I, Magalhães L, Araújo MJ, Almeida Filho ND, Aquino E, Oliveira MM. Hipertensão arterial na população adulta de Salvador (BA)-Brasil. Arq. Bras. Cardiol. 2006 dez.; 87(6):747-56.

14. Bassanesi SL, Azambuja MI, Achutti AC. Mortalidade precoce por doenças cardiovasculares e desigualdades sociais em Porto Alegre: da evidência à ação. Arq. Bras. Cardiol. 2008; 90(6):403-12.

15. Nogueira MC, Ribeiro LC, Cruz OG. Desigualdades sociais na mortalidade cardiovascular precoce em um município de médio porte no Brasil. Cad. Saúde Pública. 2009 nov.; 25(11):2321-32.

16. Melo EC, Carvalho MS, Travassos C. Distribuição espacial da mortalidade por infarto agudo do miocárdio no Município do Rio de Janeiro, Brasil. Cad. Saúde Pública. 2006 jun.; 22(6):1225-36.

17. Drumond Jr M, Barros MB. Desigualdades socioespaciais na mortalidade do adulto no município de São Paulo. Rev. Bras. Epidemiol. 1999 ago. 1;2:34-49.

18. Eyken EBBDV, Moraes CL. Prevalência de fatores de risco para doenças cardiovasculares entre homens de uma população urbana do Sudeste do Brasil. Cad. Saúde Pública. 2009; 25(1):111-23.

19. Ministério da Saúde. Plano de ações estratégicas para o enfrentamento das doenças crônicas não transmissíveis (DCNT) no Brasil 2011-2022 [Internet]. Brasília: Ministério da Saúde; 2011 [Citado 2017 jan.08]. Disponível em: <http://bvsms.saude.gov.br/bvs/publicacoes/plano acoes enfrent dent 2011.pdf $\geq$.

20. Malta DC, Santos MA, Stopa SR, Vieira JE, Melo EA, Reis AA. A cobertura da Estratégia de Saúde da Família (ESF) no Brasil, segundo a Pesquisa Nacional de Saúde, 2013. Ciênc. Saúde Coletiva. 2016 fev.; 21(2):327-38.

21. Ministério da Saúde. Informações em Saúde [Internet]. Brasília: Ministério da Saúde; 2008 [Citado 2013 fev. 05]. Disponível em: <http://www2.datasus.gov.br/DATASUS/index.php?area=02>.

22. Segi M. Cancer mortality for selected sites in 24 countries (1950-57). Sendai, Tohoku University School of Public Health; 1960.

23. Instituto Brasileiro de Geografia e Estatística (IBGE). Informações em Saúde [Internet]. Brasília: Ministério da Saúde; 2008 [Citado 2013 mar. 12]. Disponível em: <http://www2.datasus.gov.br/DATASUS/index.php?area=0206>.

24. Programa das Nações Unidas para o Desenvolvimento (PNUD). IDHM Municípios 2010 [Internet] [Citado 2013 maio 28]. Disponível em: <http://www.pnud.org.br/atlas/ranking/Ranking-IDHM-Municipios-2010.aspx>.

25. Alves MJM. Análise multivariada no estudo da dinâmica de determinantes de saúde. Rio de Janeiro: COPPE/UFRJ, Engenharia Biomédica; 1995.

26. Instituto Brasileiro de Geografia e Estatística (IBGE). Sinopse do Censo Demográfico 2010. Minas Gerais [Internet]. Rio de Janeiro: Instituto Brasileiro de Geografia e Estatística; 2010 [Citado 2017 fev. 27]. Disponível em: <http://www.censo2010.ibge.gov.br/sinopse/index.php?uf=31>. 
27. Go AS, Mozaffarian D, Roger VL, Benjamin EJ, Berry JD, Borden WB, et al. Heart disease and stroke statistics-2013 update. Circulation. 2013 jan. 1;127(1).

28. Fernandes AS, Seclen-Palacin J. Experiências e desafios da atenção básica e saúde da família: caso Brasil. Brasília: Organização Pan-Americana da Saúde; 2004.

29. Medeiros CR, Meneghel SN, Gerhardt TE. Desigualdades na mortalidade por doenças cardiovasculares em pequenos municípios. Ciênc. Saúde Coletiva. Rio de Janeiro, RJ. 2012 nov.; 17(11):2953-62.

30. Alfradique MA, Bonolo PF, Dourado I, Lima-Costa MF, Macinko J, Mendonça CS, et al. Internações por condições sensíveis à atenção primária: a construção da lista brasileira como ferramenta para medir o desempenho do sistema de saúde (Projeto ICSAP - Brasil). Cad. Saúde Pública 2009; 25:1337-49.

31. Matos MD, Fiszman R. Estratégias de prevenção para doenças cardiovasculares e promoção de saúde. Rev. SOCERJ. 2003 abr.; 16(2):133-40.

32. Reddy KS, Yusuf S. Emerging Epidemic of Cardiovascular Disease in Developing Countries. Circulation. 1998; 97:596-601.

33. Andrade SS, Stopa SR, Brito AS, Chueri PS, Szwarcwald CL, Malta DC. Prevalência de hipertensão arterial autorreferida na população brasileira: análise da Pesquisa Nacional de Saúde, 2013. Epidemiol. Serv. Saúde. 2015 jun.; 24(2):297-304.

34. Iser BP, Stopa SR, Chueiri PS, Szwarcwald CL, Malta DC, Monteiro HO, et al. Prevalência de diabetes autorreferido no Brasil: resultados da Pesquisa Nacional de Saúde 2013. Epidemiol. Serv. Saúde. 2015 jun.; 24(2):305-14.

35. Souza MFM, Rocha FM, Malta DC, Morais OLM, Silva JB Jr. Epidemiologia das doenças do aparelho circulatório no Brasil: uma análise da tendência da mortalidade. Rev. Soc. Cardiol. Est. São Paulo. 2006; 16(1):48-62.

Submissão: julho de 2017.

Aprovação: maio de 2018. 